\title{
Optical and Electrical Spectrum Analysis of Optoelectronic Devices
}

\author{
Ning Hua Zhu, Wei Chen, Wei Li, Li Xian Wang, \\ Xiao Qiong Qi and Bang Hong Zhang \\ Institute of Semiconductors, Chinese Academy of Sciences \\ P. R. China
}

\section{Introduction}

\subsection{Introduction of optical and electrical spectrum analysis}

Electrical/optical (E/O) and optical/electrical (O/E) devices constitute a cascaded network as shown in Fig. 1.1. Four scattering parameters $\left(S_{11}, S_{12}, S_{21}\right.$ and $\left.S_{22}\right)$ of the cascaded network can be easily obtained using a calibrated microwave vector network analyzer (VNA). However, to characterize the individual E/O or O/E device in such a cascaded network, a calibrated O/E or E/O device is required. Even though, other characteristics in both optical and electrical domains can not be extracted from these four scattering parameters. Optical and electrical spectrum analyses with the combination of the VNA sweeping techniques can provide simple and effective approaches to characterize highspeed $\mathrm{E} / \mathrm{O}$ and $\mathrm{O} / \mathrm{E}$ devices.

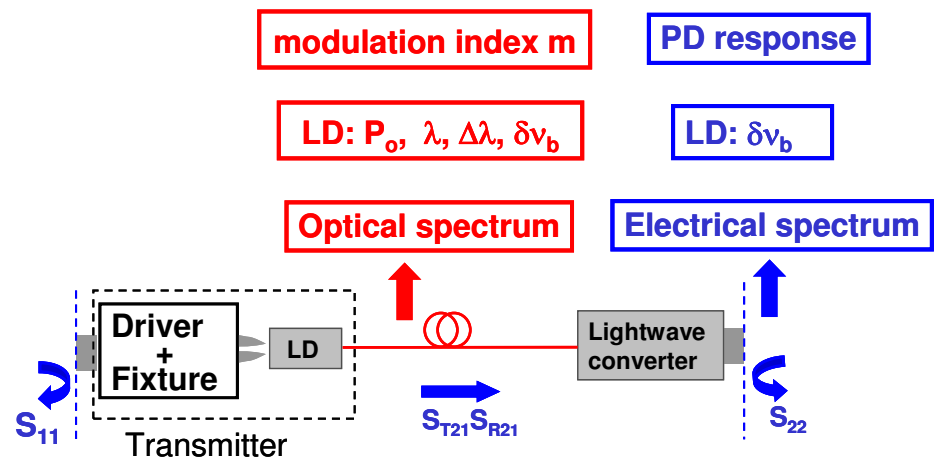

Fig. 1.1. Schematic diagram of optical and electrical spectrum analyses for characterizing optoelectronic devices

From Fig. 1.1, it can be seen that the optical spectrum emitted from the light transmitter and the electrical spectra at the electrical output port of the lightwave converter can be measured by using an optical spectrum analyzer (OSA) and an electrical spectrum analyzer (ESA), respectively. Many important characteristics of optoelectronic devices can be obtained from these spectra. For instance, optical power, wavelength, mode interval and linewidth can be 
extracted from the optical spectra. Other properties, which are usually measured in the electrical domain, can also be obtained, such as frequency responses of broadband optical external modulators (Yan et al., 2005) and directly modulated semiconductor lasers (Henery et al., 1989; Bjerkan et al., 1996), chirp parameters of light transmitters (Villafranca et al., 2005) and optical injection properties of semiconductor lasers (Simpson et al., 1997). Since optical receiver is not required in the optical spectrum analysis, there is no upper limit in the measurement of frequency responses. Moreover, this technique is simple to implement because only one OSA is used in the measurement. Nevertheless, the resolution of an OSA is usually several tens of picometers, which limits the application of this technique in frequency response measurement at lower modulation frequency.

Characteristics of optoelectronic devices, such as frequency responses of high-speed photodetectors (Zhu et al., 2006), tuning characteristics of tunable lasers, time-resolved optical spectrum (Yu \& O'Dowd, 2002), chirp parameters and fibre dispersion coefficients (Kowalski et al., 1999), can be precisely measured by means of electrical spectrum analysis. Compared to the optical spectrum analysis, electrical spectrum analysis is more accurate in analysis of optical spectral properties. For instance, in the optical spectral linewidth measurement, a resolution of $1 \mathrm{mHz}$ can be achieved theoretically (Zhu et al., 2007).

According to the above discussions, optical and electrical spectrum analyses extend the applications in optoelectronic device characterization. Meanwhile, the combination of these two techniques would bring a new perspective in optoelectronic device designing and testing. This chapter is organized as follows. In Section 2, the techniques in characterizing optoelectronic devices in the electrical domain are introduced. In Section 3, the techniques in characterizing optoelectronic devices in the optical domain are introduced. In Section 4, the optical spectrum analysis is used to investigate the optical multi-beam injected semiconductor lasers, followed by the conclusion and prospect in Section 5 .

\section{Characterization based on electrical spectrum analysis}

Along with the development of the used optical spectrum analyzer communication system, characterizing optoelectronic devices accurately and rapidly becomes more and more important. In the past decade, reasearchers have dedicated to develop techniques based on electrcial domain ananlysis because of its high resolution. In this sectin, we will introduce some of the recent progress of these techniques.

\subsection{Spectral linewidth measurement}

The widely used optical spectrum analyzer uses a scanning diffraction grating as frequency selective filter and its resolution is limited to tens of picoseconds $(>1 \mathrm{GHz})$, whereas, the linewidths of distributed feedback (DFB) laser and distributed Bragg reflector (DBR) laser are of the order of $10 \mathrm{MHz}$ and that of fiber laser is even narrower than $1 \mathrm{kHz}$. Since it is difficult to improve the resolution bandwidth of the optical spectrum analyzer, the linewidth measurement mainly takes advantage of optical heterodyne technique. The main idea is transferring the detect domain from optical frequency to microwave frequency and calculating the optical spectral $3 \mathrm{~dB}$ linewidth by measuring the electrical spectral linewidth and analyzing the spectral lineshape. Recently Agilent, Tektronix and R\&S companies all have electrical spectrum analyzer with the resolution bandwidth lower than $10 \mathrm{~Hz}$ and the real-time electrical spectrum analyzer improves the resolution to $1 \mathrm{mHz}$. Theoretically, optical heterodyne method can be used to measure the laser linewidth of the order of millihertz. 
In the following we will introduce several methods of linewidth measurement by using electrical spectrum analyzer.

1. Dual-beam optical heterodyne method

This method needs a stable and linewidth-known reference tunable laser. For two beams of Lorentzian lineshapes, the linewidth of their beat signal is the sum of that of the two optical spectra. For both Gaussian lineshapes, the squared linewidth of the beat signal is the sum of the squared linewidths of the two beams. So, if the linewidth of the reference laser is far narrower than that of the under test laser, the linewidth of the beat signal can be estimated to be that of the under test laser.

The dither between the reference laser and the under test laser limits the measurement accuracy.

2. Self-homodyne method

The under test lightwave is injected to a Mazh-Zehnder interferometer and split into two unequal arms. The beat signal is at zero frequency. If the delayed fiber line is much longer than the coherence length, the measured $3 \mathrm{~dB}$ linewidth of the self-homodyne signal is two times of that of the under test laser.

In this method, the amplitude noise and phase noise are mutually overlapped. The important linewidth information is, however, connected with the phase noise.

3. Self-heterodyne method

To distinguish the amplitude and phase noise spectra, it is better to shift the beat signal to none-zero frequency. The simplest way is to add an acousto-optical frequency shifter to one arm. Thus, the beat signal could be detected by a standard detector, but the inducing of the shifter will cause an optical loss.

In practice, frequency and polarization fluctuations of the under test laser will affect the linewidth measurement. So we can say that it is hard to accurately measure the laser linewidth when frequency and polarization jitters are serious.

\subsection{Frequency response measurement for high-speed photodetectors}

Wide-bandwidth photodetectors are essential devices for very high-speed optical transmission and signal processing systems. Many methods for measuring the frequency responses of photodetectors have been proposed, such as the swept frequency method (Humphreyst, 1989), the pulse spectrum analysis method (Shao \& Gallawa, 1986), the interferometric FM sideband method (Ohishi et al., 1989) the optical heterodyne detection method (Hawkins et al., 1991), the intensity noise technique (Baney et al., 1994).

\subsubsection{Improved self-heterodyne method}

Among these methods, optical heterodyne method has drawn more and more attention of engineers due to its advantages. In the conventional optical heterodyne method, two lasers with the requirement of precise wavelength (less than $0.5 \mathrm{~nm}$ ) and polarization mode matching are utilized. One of the lightwave sources is lasing at fixed wavelength and the other one is tuned continuously over the whole measurement range. However, the wavelength stability of both lasers is rigorous because any slight drifts in the operating condition of the lasers can cause unwanted frequency and power fluctuations which affect the accuracy of measurements of frequency responses. All of these problems can be avoided by using the self-heterodyne method (Hou et al., 1989).

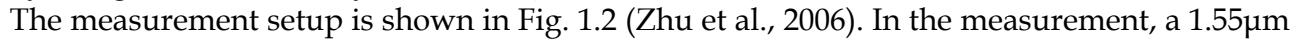
four-section DBR laser is used. The two Bragg sections are shorted. The output wavelength 
is tuned by the current applied to the phase section and is measured by an OSA. With the application of a square wave voltage, the tunable DBR is operated in two states corresponding to high and low levels of a square-wave voltage. The low level of the square wave voltage is fixed, corresponding to a fixed optical wavelength. The high level of the square wave voltage is tuned, producing a tunable optical wavelength. Therefore, the laser switches periodically between two optical wavelengths.

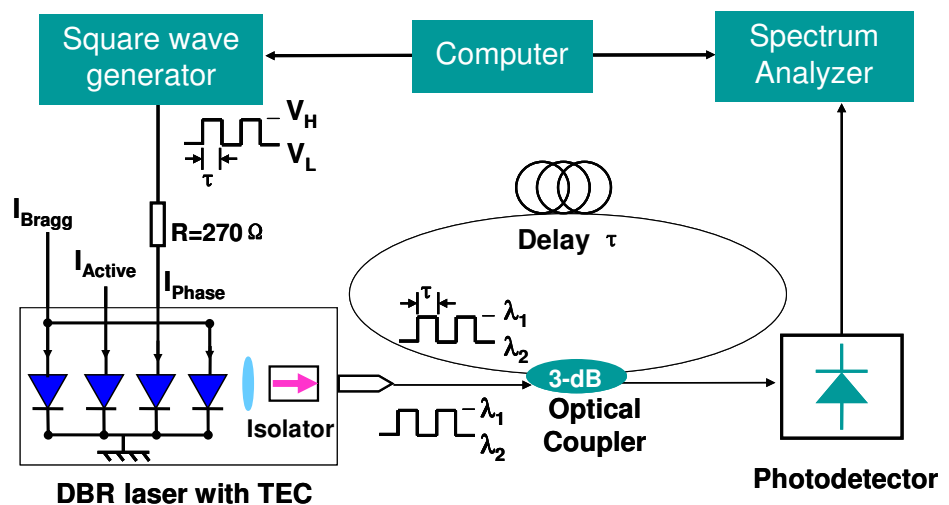

Fig. 1.2. Measurement setup of self-heterodyne method for characterizing frequency responses of photodetectors

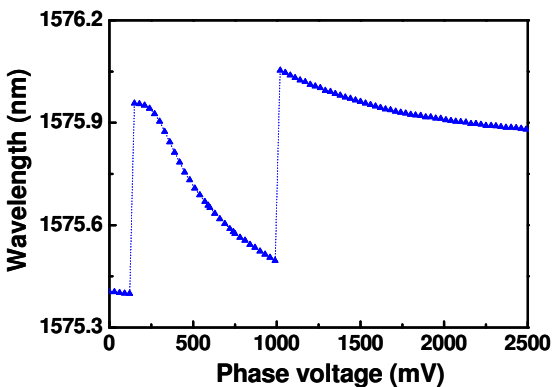

(a)

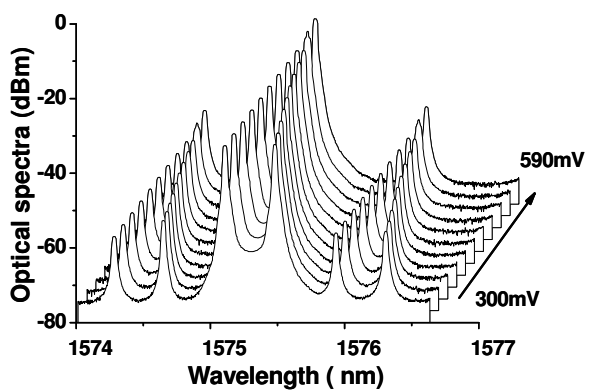

(b)

Fig. 1.3. (a) Measured optical wavelength and (b) spectra as functions of the phase voltages

Part of the optical power is delayed by half of the square wave voltage period after passing through the fiber ring interferometer. The delayed and undelayed light waves beat in the photodetector to generate a microwave signal. The center frequency of the beat signal equals to the wavelength difference between the two wavelength states. The tuning properties as functions of the phase voltage are shown in Fig. 1.3. It can be seen that the wavelength continuously decreases with the applied phase voltage, and the frequency sweeps from DC to tens of gigaherz. Fig. 1.3(b) shows the optical spectra when a square-wave voltage is applied, in which the high voltage level is fixed, and the low voltage level varies The experimental results are shown in Fig. 1.4. The measured linewidths are between 30 $\mathrm{MHz}$ and $100 \mathrm{MHz}$. The trace of the beat signal peaks represents the measured frequency 
responses of the photodetector. However, this response still includes the influences of the fluctuations of the optical power and the linewidths of the beat signals.
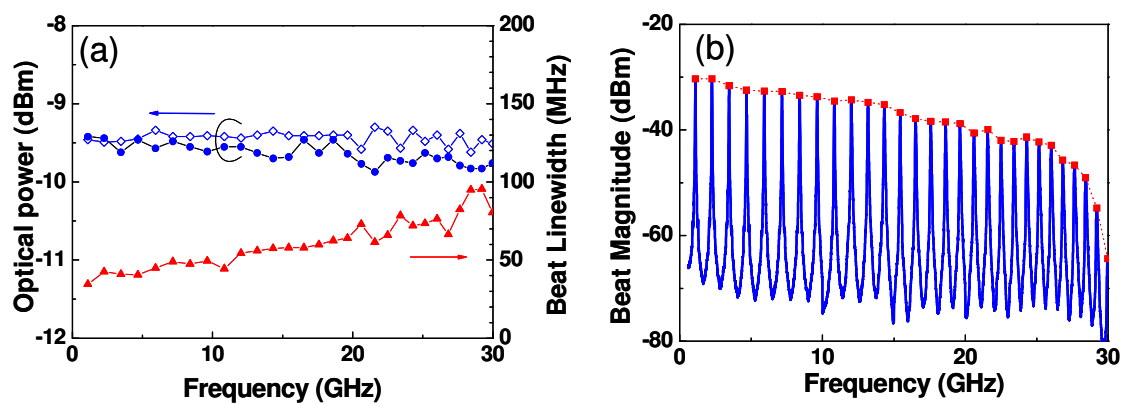

Fig. 1.4. (a) Measured linewidths of the beat signals and optical peak powers of two wavelengths. (b) Measured magnitudes of the beat signals, where $V_{L}$ is fixed

Assuming the line shape is Lorentian, the peak power of the beat spectrum measured using an ESA can be expressed as (Zhu et al., 2006):

$$
\begin{aligned}
P_{b}\left(v_{b}\right) & \propto\left(\frac{e \eta}{h v}\right)^{2} \cdot E_{1}{ }^{2} \cdot E_{2}{ }^{2} \cdot R_{L} \cdot F^{2}\left(v_{b}\right) \cdot \int_{v_{b}-B / 2}^{v_{b}+B / 2} \frac{\delta v_{b}}{2 \pi\left\{\left(v-v_{b}\right)^{2}+\left(\frac{\delta v_{b}}{2}\right)^{2}\right\}} d v \\
& \propto \frac{2}{\pi} \cdot\left(\frac{e \eta}{h v}\right)^{2} \cdot E_{1}{ }^{2} \cdot E_{2}{ }^{2} \cdot R_{L} \cdot F^{2}\left(v_{b}\right) \cdot \arctan \frac{B}{\delta v_{b}}
\end{aligned}
$$

where $h$ is the photon energy, $e$ is the electron charge, $\eta$ is quantum efficiency, $E_{1}$ and $E_{2}$ are the optical fields corresponding to the voltages $V_{H}$ and $V_{L}$ applied to the phase section, respectively. $F\left(v_{b}\right)$ is the frequency response coefficient of the photodetector, $B$ and $R_{L}$ are resolution bandwidth of the ESA and the input impedance, respectively. Therefore, the frequency response of the photodetector can be obtained as follows:

$$
F\left(v_{b}\right)=C \cdot\left|\frac{P_{b}\left(v_{b}\right)}{E_{1}^{2} E_{2}^{2} \arctan \left(B / \delta v_{b}\right)}\right|^{\frac{1}{2}}
$$

As the amplitudes of driving signal increase, the beat signal may not be a Lorentian line shape. In this case, the calibration should be made using the actual lineshape, and (2) becomes:

$$
F\left(v_{b}\right)=C \cdot\left|\frac{P_{b}\left(v_{b}\right)}{E_{1}^{2} E_{2}^{2} \int_{v_{b}-B_{0}}^{v_{b}+B_{0}} S_{b}(v) d v}\right|^{\frac{1}{2}}
$$

where $B_{0}$ and $S_{b}\left(v_{b}\right)$ is the $10-\mathrm{dB}$ linewidth of the beat signal and the power spectrum of the beat signal respectively. From the measurement results, on can see that, the results obtained using both sweeping methods are similar after calibration, and agree well with the Agilent data. 


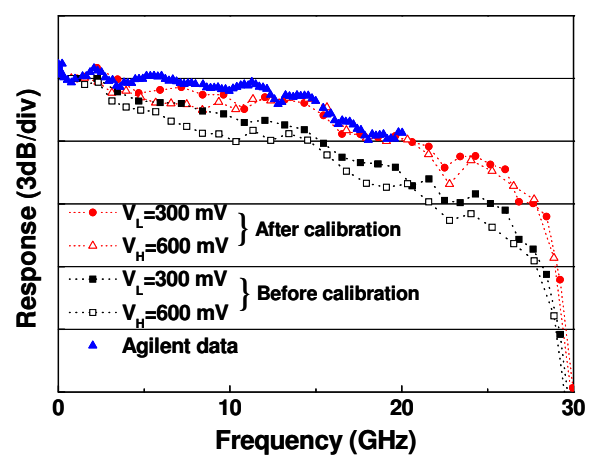

(a)

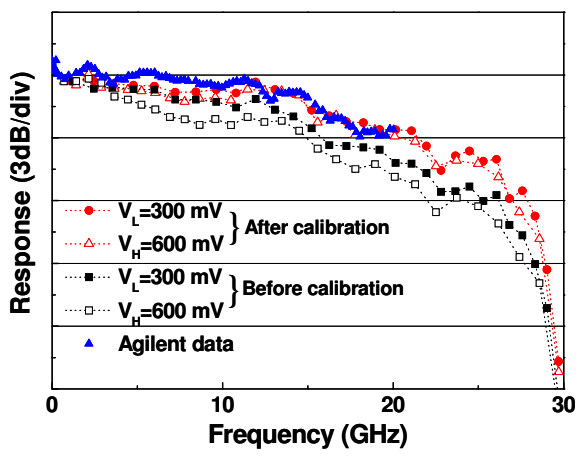

(b)

Fig. 1.5. Measured frequency responses of the photodetector from Agilent when the Bragg sections are (a) shorted and (b) biased at $12 \mathrm{~mA}$

\subsubsection{Harmonic analysis technique}

In this section, the swept frequency method for measuring the frequency response of a PD based on harmonic analysis is introduced. The schematic diagram is shown in Fig. 1.6. The output of a laser is modulated by a swept frequency RF signal via a Mach-Zehnder $\mathrm{LiNbO}_{3}$ modulator, and then detected by a photodetector. The 2nd-order harmonic of the RF signal contains information of the frequency responses and the nonlinearities of the RF source,optical modulator and PD. It has been shown that the small-signal power measuring technique can be used to measure the frequency response of the RF source and modulator (Huang \& Zhu, 2006). Therefore, by deducting the known frequency responses and the nonlinearities of the RF source and modulator, the frequency response of the photodetector can be obtained (Zhang et al., 2009).

The optical output of an $\mathrm{LiNbO}_{3}$ modulator can be written as:

$$
\begin{aligned}
P_{\text {out }}= & \frac{1}{2} P_{0}\left\{1+\cos \phi_{0} J_{0}\left(F\left(\omega_{\mathrm{m}}\right)\right)\right. \\
& +2 \cos \phi_{0} \sum_{n=1}^{+\infty}(-1)^{n} J_{2 n}\left(F\left(\omega_{\mathrm{m}}\right)\right) \cos \left(2 n \omega_{\mathrm{m}} t\right) \\
& \left.+2 \sin \phi_{0} \sum_{n=1}^{+\infty}(-1)^{n} J_{2 n-1}\left(F\left(\omega_{\mathrm{m}}\right)\right) \cos \left[(2 n-1) \omega_{\mathrm{m}} t\right]\right\}
\end{aligned}
$$

where $F\left(\omega_{m}\right)$ is the frequency response of the RF source and modulator. The detected power of 2nd harmonic can be expressed as follows, in which $M_{p}\left(\omega_{m}\right)$ denotes the frequency response of the photodetector.

$$
P_{2 \omega_{\mathrm{m}}} \propto J_{2}^{2}\left(F\left(\omega_{\mathrm{m}}\right)\right) M_{\mathrm{p}}^{2}\left(2 \omega_{\mathrm{m}}\right) \propto F^{4}\left(\omega_{\mathrm{m}}\right) M_{\mathrm{p}}^{2}\left(2 \omega_{\mathrm{m}}\right)
$$

When drive signal consists of a single tone at a low frequency $\omega_{1}$ low enough that $M_{p}\left(\omega_{1}\right)=1$, the detected power of the signal at $\omega_{1}$ is (Huang \& Zhu, 2006):

$$
P_{\omega_{1}} \propto i_{\omega_{1}}^{2} \propto F^{4}\left(\omega_{m}\right)
$$


Substituting (6) into (5), the frequency response of the PD can be easily obtained by normalizing the results. Fig. 1.7 shows the results when a $10 \mathrm{GHz}$ modulator is employed. It can be seen that the data after calibration fit well with the reference data. We can also see the measurement range is doubled using harmonic analysis method compared to that using the conventional swept frequency technique.

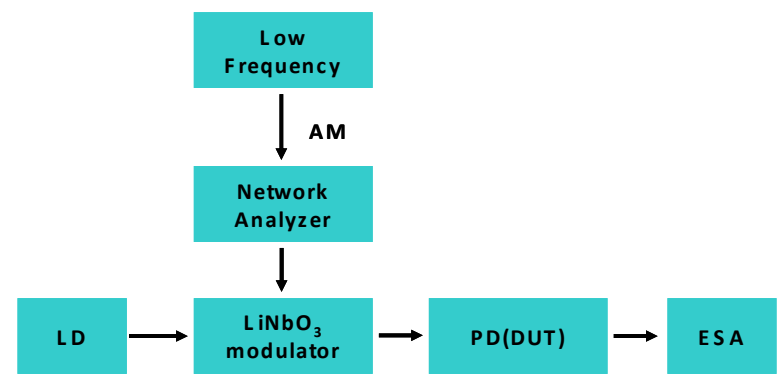

Fig. 1.6. Schematic diagram of the measurement setup

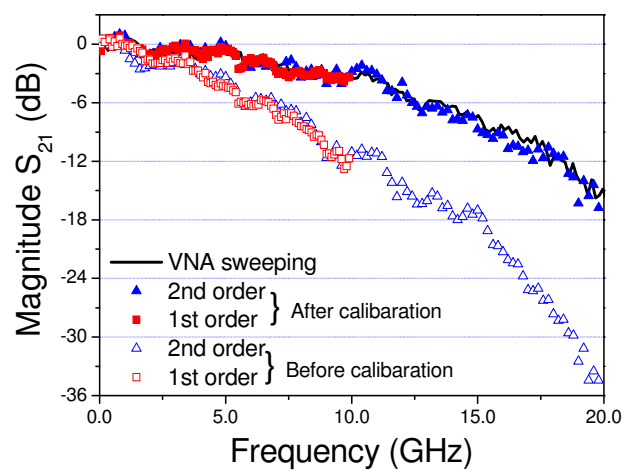

Fig. 1.7. Measurement results

\subsection{Tuning characteristics of tunable lasers measurement}

The lightwave wavelengths of semiconductor lasers vary with bias current and environment temperature. The central wavelength can be easily obtained from optical spectrum analyzer. With the bias current tuned, the wavelength variation of normal lasers is actually due to the change of junction temperature. It is the heat induced change in refractive index that leads to the changes in wavelength. This period is relatively slow and the response time is of the order of milliseconds. However, the lightwave wavelength of DBR tunable laser varies fast with phase section current, and the response time is of the order of nanoseconds. In practice, fast tunable semiconductor lasers are considered to be the essential devices in optical packet switching technique which is one of the key techniques of the next optical communication network. The prerequisite of such lasers is the ability of fast switching in the limitation of the order of nanometer. Thus, it is important to study the instantaneous properties of the tunable laser. On the one hand, by studying the instantaneous characteristics one can 
achieve the wavelength switching time and the optimum time for the wavelength-stable output. These parameters are important to judge whether a system meets requirement. On the other hand, linewidth broadening is also a dynamic process. The linewidth of the tunable laser in the tuning process is quite different from that in steady state. The linewidth broadening will induce dispersion which will deteriorate the transmission. Therefore, deeply studying the instantaneous characteristics of the tunable laser and the influence factors is critical to the assessment and improvement of device properties.

The resolution of optical spectrum analyzer is of the order of $0.01 \mathrm{~nm}$, which can not meet the requirement of measuring the dynamic process, whereas by combining optical and electrical spectrum analyses we can easily solve this problem. We introduce two methods to measure the tuning speed. One is using optical filter to filter out one wavelength. Then after detected by a photoelectric detector, the electric signal is observed using an oscilloscope. Another method is using a reference laser and taking advantage of optical heterodyne technique to generate a microwave beat signal. By detecting the beat signal's varying speed one can obtain the optical tuning speed. The time response of the beat signal is detected using "zero span" function of the electrical spectrum analyzer.

\subsection{Time-resolved optical spectrum measurement}

In many applications, such as the transmission of AM and FM optical signal, channel switch in FDM system, the transient characteristics of laser wavelength should be always taken into account. Researchers have paid much attention in developing techniques to measure the time-resolved optical spectra. One of them (Bergano, 1988) is based on an optical filter that is used as a discriminator. The absorption coefficient of the filter is a function of the optical wavelength, so that the variation of wavelength can be converted into intensity variation. In this technique, the pass band of the optical filter is relatively wide. Another method ( $\mathrm{Yu} \&$ $\mathrm{O}^{\prime}$ Dowd, 2002) also uses an optical filter, but its pass band is narrow and only one wavelength can be selected. After passing through this filter, an oscilloscope is used to observe the intensity variation of the output. Commonly the signal under test is periodic, so that when the frequency centre of the optical filter is changed, optical signals with other frequencies can be measured. By combing all the data, the time-resolved optical spectrum can be obtained. The schematic setup of these two methods is shown in Fig. 1.8(a).

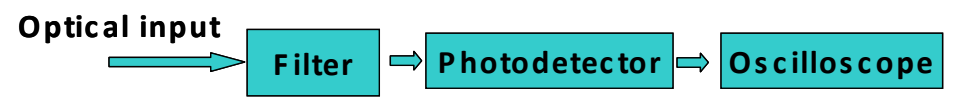

(a)

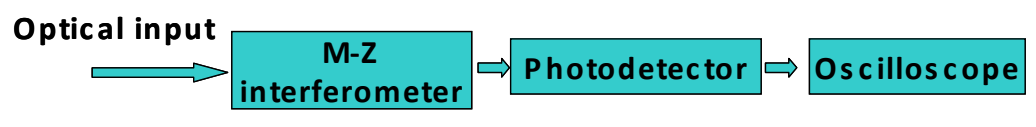

(b)

Fig. 1.8. Schematic setup for measuring time-resolved optical spectra

There is also a method that uses self-heterodyne technique (Joseph \& Sadot, 2004), which measuring the time-resolved optical spectra in electrical domain. The input optical signal is divided into two branches, one of which is delayed. Then the beat signal of the two signals 
is measured, which is equivalent to obtain the autocorrelation function. After some calculation, the time-resolved optical spectrum can be deduced. The schematic setup is shown in Fig. 1.8(b).

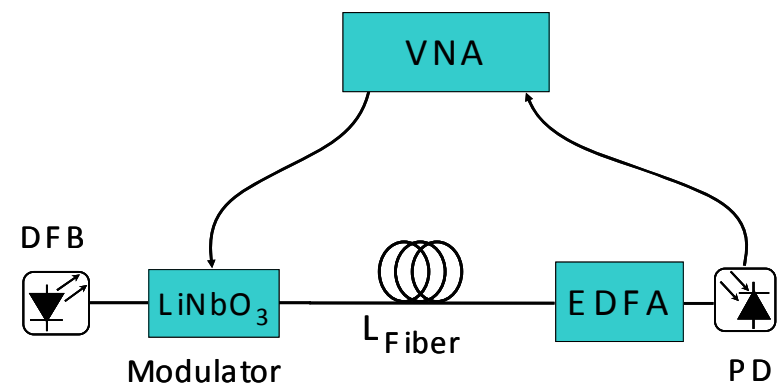

Fig. 1.9. Experiment setup for measuring the chirp parameter and fibre dispersion

\subsection{Measurement of chirp parameter and fiber dispersion}

Almost all of the laser sources, including directly modulated semiconductor laser, electroabsorption modulator, $\mathrm{LiNbO}_{3}$ modulator, have the characteristic of frequency chirp. The chirp parameter indicates the relationship between the intensity variation and the phase variation:

$$
\delta \phi=\frac{\alpha \delta \phi}{2 I}
$$

$I$ is the optical intensity, $\Phi$ denotes the phase of the laser, $a$ is the chirp parameter.

The chirp of the laser source together with the chromatic dispersion of single mode fibre (SMF), which is simply a variation in the speed of propagation of a lightwave with a wavelength, can severely affect the performance of optical communication systems based on fibre. Consequently, characterizing these two parameters accurately plays an important role in designing fibre based communication system.

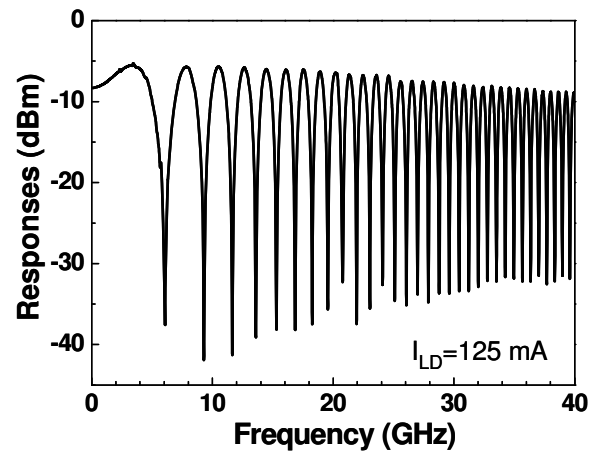

(a)

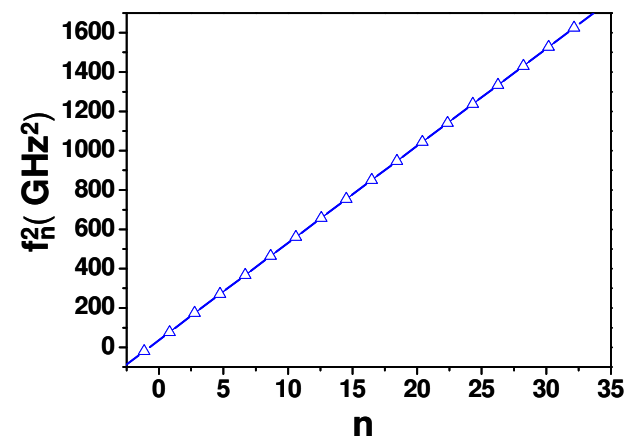

(b)

Fig. 1.10. (a) Calibrated fibre transfer function. (b) The measured results according to (9) 
By using the sweep method based on VNA, the small-signal chirp parameter and the dispersion coefficient can be measured simultaneously (Kowalski et al., 1999). The fibre transfer function can be expressed as follows:

$$
I_{f} \propto\left|\cos \left(\frac{\pi \lambda^{2} D L f^{2}}{c}+\arctan \alpha\right)\right|
$$

Firstly, the long fibre under test is replaced by a short length of fibre and the optical-tooptical calibration is implemented to remove the impact induced by the VNA, the modulator and the photodetector. Then the scattering parameter $S_{21}$ of the long fibre is measured. The square of the frequency $f_{n}$ according to the nth zero pole is proportional to the reciprocal of the dispersion coefficient:

$$
f_{n}^{2} L=\frac{c}{2 D \lambda^{2}}\left(1+2 n-\frac{2}{\pi} \arctan \alpha\right)
$$

The measurement setup is shown in Fig. 1.9. The results are shown in Fig. 1.10. As shown in Fig. 1.10(b), we can obtain the fibre dispersion coefficient and the chirp parameter from the slope and the intercept respectively according to (9). This method is accurate and simple. The measurement uncertainty is about \pm 0.1 .

\section{Characterization based on optical spectrum analysis}

In this section, we introduce the techniques for characterizing optoelectronic devices based on optical spectrum analysis, which can be easily carried out with simple experimental setups. The frequency responses of the lasers or modulators can be extracted from the measured optical spectrum.

\subsection{Frequency responses of broadband modulators}

We use sideband calculating method which is proposed by Auracher and Keil for the first time (Auracher \& Keil, 1980), and developed by Shi and Willner et al. (Shi et al., 2003; Yan et al., 2005; Yan et al., 2003; Kawanishi et al., 2001; Oikawa et al., 2003), to obtain the frequency responses of modulators based on optical spectrum analysis. The sidebands produced by direct or external modulation can be observed by OSA. The frequency modulation index can be calculated from the intensities of the optical sidebands, and then the frequency response of the modulator can be obtained. For a Mach-Zehnder modulator, the output optical field can be written as:

$$
E_{\mathrm{o}}=\frac{E_{\mathrm{i}}}{2} \mathrm{e}^{\mathrm{j} \omega_{0} t}\left(\mathrm{e}^{\mathrm{j} \Phi_{1}} \mathrm{e}^{\mathrm{j} \eta_{1} U_{\mathrm{m}} \cos \omega_{\mathrm{m}} t}+\mathrm{e}^{\mathrm{j} \Phi_{2}} \mathrm{e}^{\mathrm{j} \eta_{2} U_{\mathrm{m}} \cos \omega_{\mathrm{m}} t}\right)
$$

where $U_{m} \cos \omega_{m} t$ is the applied voltage, $\omega_{0}$ is the frequency of the lightwave, and $\Phi_{1} \cdot \Phi_{2}$, $\eta_{1} \cdot \eta_{2}$ are the inherent phase shifts and the phase modulation indices in two arms, respectively.

Assuming that the incident optical field is equally split into two beams, and the applied electrical fields in the two arms are with the same phase, the above equations can be expanded into Fourier series by Bessel function as follows: 


$$
E_{\mathrm{o}}=\frac{E_{\mathrm{i}}}{2} \mathrm{e}^{\mathrm{j} \omega_{0} t} \sum_{n=-\infty}^{\infty} \mathrm{j}^{n}\left[\mathrm{e}^{\mathrm{j} \Phi_{1}} \mathrm{~J}_{n}\left(\eta_{1} U_{\mathrm{m}}\right)+\mathrm{e}^{\mathrm{j} \Phi_{2}} \mathrm{~J}_{n}\left(\eta_{2} U_{\mathrm{m}}\right)\right] \times \mathrm{e}^{\mathrm{j} n \omega_{\mathrm{m}} t}
$$

From (11), we can see that the output optical field includes not only the carrier component $\omega_{0}$, but also the infinite sidebands components $\omega_{0}+n \omega_{m}$. The optical intensity of each sideband can be expressed as:

$$
I\left(\omega_{0}+n \omega_{\mathrm{m}}\right)=\frac{I_{\mathrm{i}}}{4}\left[\mathrm{~J}_{n}^{2}\left(\eta_{1} U_{\mathrm{m}}\right)+\mathrm{J}_{n}^{2}\left(\eta_{2} U_{\mathrm{m}}\right)+2 \mathrm{~J}_{n}\left(\eta_{1} U_{\mathrm{m}}\right) \mathrm{J}_{n}\left(\eta_{2} U_{\mathrm{m}}\right) \cos \left(\Phi_{1}-\Phi_{2}\right)\right]
$$

For M-Z interference intensity modulator, we have $\eta_{1}=-\eta_{2}=\eta$, and (12) can be rewritten as:

$$
I\left(\omega_{0}+n \omega_{\mathrm{m}}\right)=\frac{I_{\mathrm{i}}}{2} \mathrm{~J}_{n}^{2}\left(\eta U_{\mathrm{m}}\right)\left[1+(-1)^{n} \cos \left(\Phi_{1}-\Phi_{2}\right)\right]
$$

Under small-signal modulation, the Bessel function can be expanded as Taylor series. That is:

$$
\mathrm{J}_{1}\left(\eta U_{\mathrm{m}}\right) \approx \frac{\eta U_{\mathrm{m}}}{2}, \mathrm{~J}_{2}\left(\eta U_{\mathrm{m}}\right) \approx \frac{\left(\eta U_{\mathrm{m}}\right)^{2}}{8}
$$

The modulation index can be obtained from the ratio between the intensities of the 1st and 2nd harmonic components,

$$
R_{2,1}(\eta)=\frac{\mathrm{J}_{2}^{2}\left(\eta U_{\mathrm{m}}\right)}{\mathrm{J}_{1}^{2}\left(\eta U_{\mathrm{m}}\right)}=\frac{I\left(\omega_{0} \pm 2 \omega_{\mathrm{m}}\right)}{I\left(\omega_{0} \pm \omega_{\mathrm{m}}\right)}=\frac{1}{16}\left(\eta U_{\mathrm{m}}\right)^{2}
$$

These two intensities can be obtained from the measured optical spectra.

\subsection{Frequency response of directly modulated lasers}

Here we introduce the optical spectrum analysis method for frequency response measuring of the directly modulated laser in detail. When a single-mode laser is modulated at $\omega_{\mathrm{m}}$, the electrical field can be written as (Henery et al., 1989; Bjerkan et al., 1996)

$$
E(t)=E_{0} \sqrt{1+m \cos \left(\omega_{m} t\right)} \mathrm{e}^{\mathrm{i}\left[\omega_{0} t+\beta \sin \left(\omega_{m} t+\theta\right)\right]}
$$

where $E_{0}$ is the optical intensity without modulation, $m$ is the intensity modulation index, $\omega_{m}$ is the angular frequency, $\beta$ is the frequency modulation index, $\theta$ is the phase difference between frequency modulation and intensity modulation.

Assuming that the intensity modulation index $m<<1$, the electrical intensity can be expanded as:

$$
E(t)=E_{0} \sum_{p=-\infty}^{+\infty} \mathrm{e}^{\mathrm{i}\left[\omega_{0} t+p\left(\omega_{m} t+\theta\right)\right]} \times\left\{\mathrm{J}_{p}(\beta)+\frac{m}{4}\left[\mathrm{~J}_{p-1}(\beta) \mathrm{e}^{-\mathrm{i} \theta}+\mathrm{J}_{p+1}(\beta) \mathrm{e}^{\mathrm{i} \theta}\right]\right\}
$$

Hence the ratio between the intensities of 1 st sideband and the carrier can be written as:

$$
I_{+1} / I_{0}=\mathrm{J}_{1}^{2}(\beta)\left(1+\frac{m}{\beta} \cos \theta\right) / \mathrm{J}_{0}^{2}(\beta)
$$




$$
I_{-1} / I_{0}=\mathrm{J}_{1}^{2}(\beta)\left(1-\frac{m}{\beta} \cos \theta\right) / \mathrm{J}_{0}^{2}(\beta)
$$

Then, we have

$$
\mathrm{J}_{1}^{2}(\beta) / \mathrm{J}_{0}^{2}(\beta)=\left(I_{+1}+I_{-1}\right) /\left(2 \times I_{0}\right)
$$

When the frequency modulation index $\beta<<1$, the equation above can be further simplified as:

$$
\mathrm{J}_{1}^{2}(\beta) / \mathrm{J}_{0}^{2}(\beta) \approx 0.25 \beta^{2}
$$

It has been shown that $(21)$ is accurate enough when $\beta<0.6$. Since the optical power of the carrier and sidebands can be easily observed from the optical spectrum, the frequency modulation index $\beta$ can be solved from the above equation. Once $\beta$ is determined, intensity modulation index $m$ can be obtained from the following equation (Schimpe et al., 1986),

$$
m\left(\omega_{m}\right)=\frac{2 \beta\left(\omega_{m}\right)}{\alpha \sqrt{1+\left(\frac{\omega_{\mathrm{g}}}{\omega_{m}}\right)^{2}}}
$$

where $a$ is the chirp parameter, namely linewidth enhancement factor, and $\omega_{g}$ is the characteristic angular frequency. For a given laser, $\omega_{g}$ usually varies in a small range. Under small-signal modulation, $a$ is a constant. For example, for a typical $1.55 \mu \mathrm{m}$ InGaAsP DFB laser, $a$ is between 2 to 3 , and $f_{g}\left(\omega_{g} / 2 \pi\right)$ is about $2.1 \mathrm{GHz}$ (Petermann 1991). Hence, we can get the frequency response of the laser from the measured optical intensities of the carrier and sidebands.

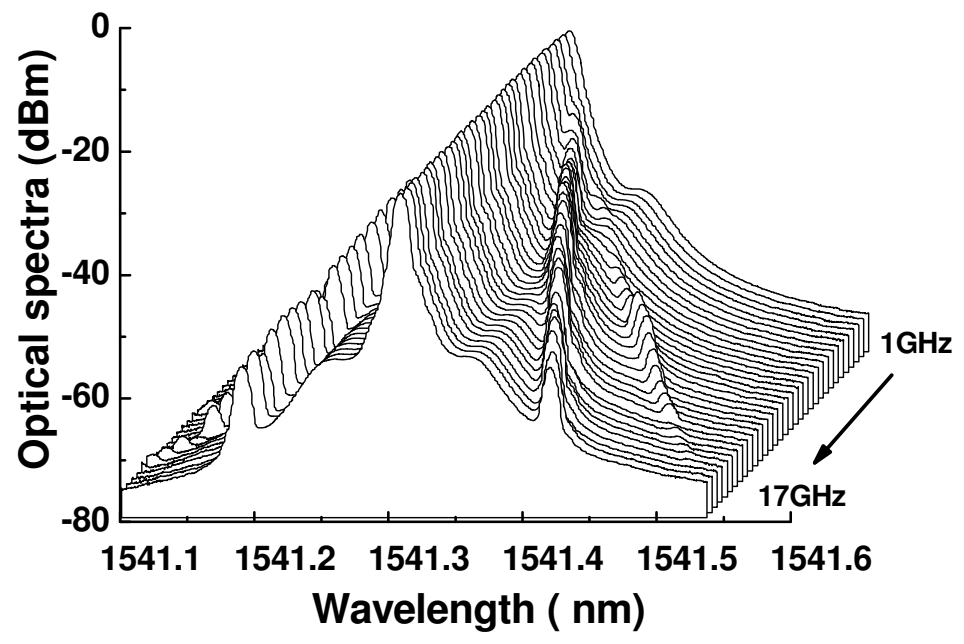

Fig. 1.11. Optical spectra at different modulation frequencies 
Fig. 1.11 shows the measured optical spectra. Since the amplitude of the modulation signal is far lower than the dynamic range of the laser, it can be considered as the case of small signal modulation.

The frequency modulation index $\beta$ solved from the optical spectra under different modulation frequencies is depicted in Fig. 1.12(a).

The amplitude modulation index calculated from (22) is plotted in Fig. 1.12(b). The frequency response obtained using sweeping method is also given in Fig. 1.12(b) for comparison, from which we can see that a good agreement has been obtained.

For conventional sweeping method using a VNA, a high speed photodetector is required, whereas, the optical spectrum analysis method is simple, and there is no need to use a calibrated phtodetector.

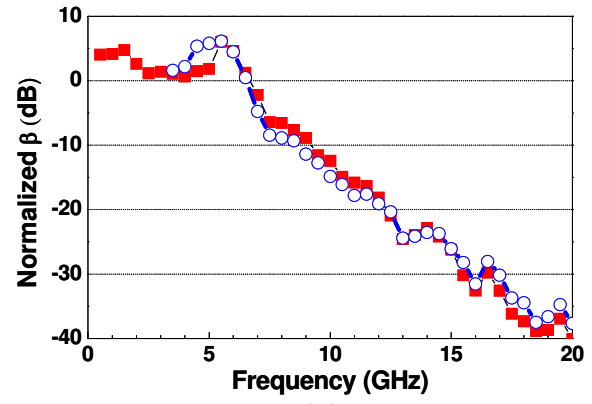

(a)

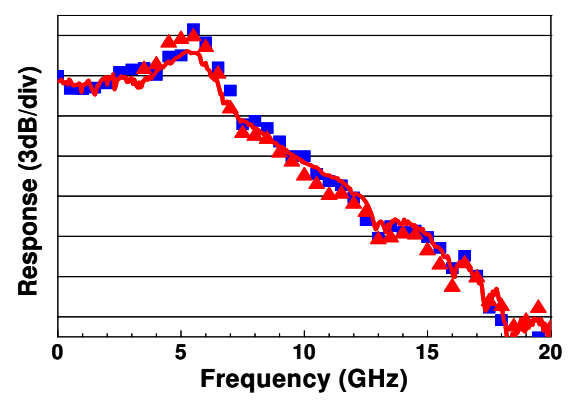

(b)

Fig. 1.12. (a)Normalized FM-modulation index and (b) frequency responses of the DFB laser

\subsection{Chirp parameters of directly modulated lasers}

The modulated optical spectrum of a laser comprises a series of sidebands with their frequencies at $\omega=\omega_{0}+n \omega_{m}$, where $n$ is an integer. As shown in Fig. 1.11, as the semiconductor laser's modulation frequency increases, the wavelength detuning between its optical sidebands increases.

In the case of small signal modulation, the optical power of the main peak, 1st order sideband and 2nd order sideband are determined by the following equations (Shi et al., 2003; Kruger et al., 1995),

$$
\begin{gathered}
I_{0}=E_{0}^{2}\left[\mathrm{~J}_{0}^{2}(\beta)+m^{2} \mathrm{~J}_{1}^{2}(\beta)\right] \\
I_{1}=E_{0}^{2}\left[\mathrm{~J}_{1}^{2}(\beta)+\left(m^{2} / 4\right)\left(\mathrm{J}_{2}(\beta)-\mathrm{J}_{0}(\beta)\right)^{2}\right] \\
I_{2}=E_{0}^{2}\left[\mathrm{~J}_{2}^{2}(\beta)+\left(m^{2} / 4\right)\left(\mathrm{J}_{3}(\beta)-\mathrm{J}_{1}(\beta)\right)^{2}\right]
\end{gathered}
$$

where $E_{0}$ is the amplitude of the optical field; $\beta$ is the frequency modulation index; $J_{n}(\beta)$ is the first kind Bessel function. The sideband powers are the average values of the upper and lower sidebands, that is, $I_{1}=\left(I_{+1}+I_{-1}\right) / 2 ; I_{2}=\left(I_{+2}+I_{-2}\right) / 2$.

According to (23)-(25), FM and IM modulation indices can be obtained by solving the following equations, 


$$
\begin{gathered}
\xi=\frac{I_{1}}{I_{0}}=\frac{\mathrm{J}_{1}^{2}(\beta)+\left(m^{2} / 4\right)\left[\mathrm{J}_{2}(\beta)-\mathrm{J}_{0}(\beta)\right]^{2}}{\mathrm{~J}_{0}^{2}(\beta)+m^{2} \mathrm{~J}_{1}^{2}(\beta)} \\
\zeta=\frac{I_{1}^{2}}{I_{0} I_{2}}=\frac{\left[\mathrm{J}_{1}^{2}(\beta)+\left(m^{2} / 4\right)\left(\mathrm{J}_{2}(\beta)-\mathrm{J}_{0}(\beta)\right)^{2}\right]^{2}}{\left(\mathrm{~J}_{0}^{2}(\beta)+m^{2} \mathrm{~J}_{1}^{2}(\beta)\right)\left[\mathrm{J}_{2}^{2}(\beta)+\left(m^{2} / 4\right)\left(\mathrm{J}_{3}(\beta)-\mathrm{J}_{1}(\beta)\right)^{2}\right]}
\end{gathered}
$$

For directly modulated laser, the relation between FM and IM indices can be expressed as (Schimpe et al., 1986)

$$
\frac{\beta}{m}=\frac{\alpha}{2} \sqrt{1+\left(\frac{\omega_{\mathrm{g}}}{\omega_{m}}\right)^{2}}
$$

where $\alpha$ is the chirp parameter, $\omega_{g}$ is the characteristic angular frequency. For a high-speed semiconductor laser, the typical value of $\omega_{g}$ is $0 \sim 3 \mathrm{GHz}^{*} 2 \Pi$. If the modulation frequency $\omega_{m}>>\omega_{g},(27)$ can be simplified as:

$$
\alpha=2 \beta / m
$$

When $\omega_{m}>5 \omega_{g}$, the (27) has an error less than $2 \%$. Using (26), the chirp parameter can be obtained by measuring the optical powers of the sidebands. However, (26) does not have analytic solutions, so the graphic solution is introduced.

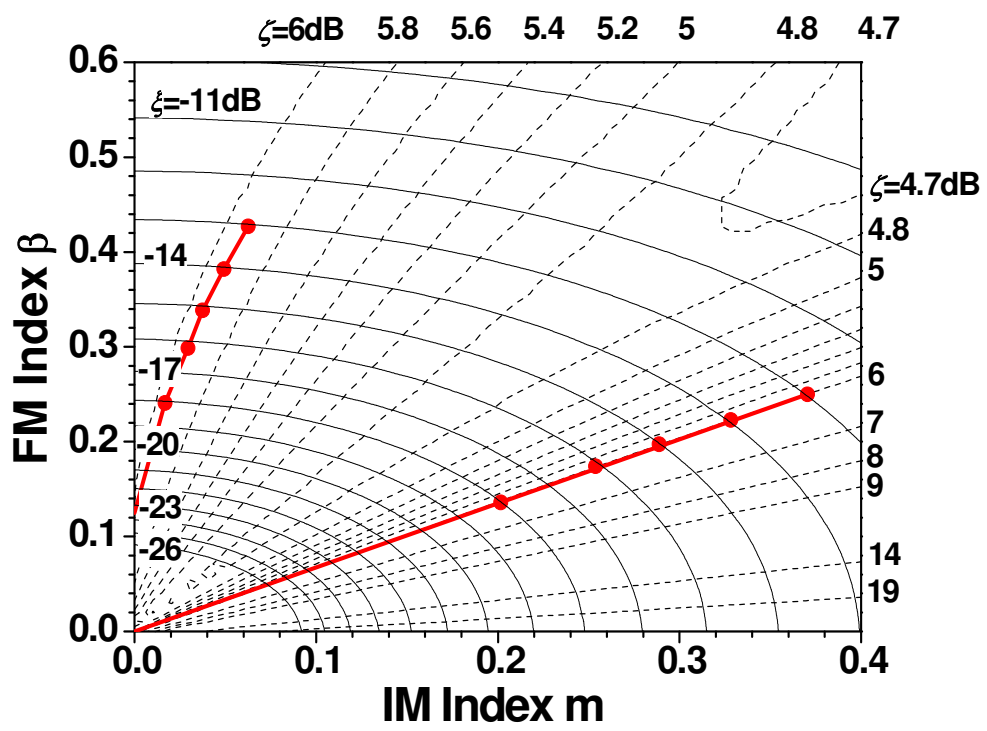

Fig. 1.13. Contour diagram for the graphic solution of FM and IM indices, where the solid line indicates $\xi$ and the dashed line indicates $\zeta$

Fig. 1.13 shows the contour diagram of $\xi$ and $\zeta$, with FM and IM indices as two orthogonal axes. The value of $\xi$ increases when the IM index increases. A straight line passing through 
the origin can be obtained by measuring $\xi$ and $\zeta$ at different modulation powers, which are defined as the RF power applied to the laser. According to (28), the slope of the straight line is $\alpha / 2$ at high modulation frequencies. Hence, the chirp parameter $a$ of the laser can be extracted from the graph. As shown in Fig. 1.13, there might be two solutions for a single measurement. Considering there is a linear relationship between $\beta$ and $m$ according to (28), the straight line which passes through the origin indicates the right solution.

For lasers with high chirp parameters, the straight line appears in the upper left part of the contour diagram. While for lasers with low chirp parameters, the line locates in the lower right part of the diagram. According to (26) and Fig. 1.13, the modulation power should be large enough to ensure the second sideband of the laser in the dynamic range of the OSA. Besides, the measurement should be in the case of small-signal modulation, so $\xi$ should be lower than $-10 \mathrm{~dB}$. As a result, the modulation power should meet the above two conditions.

Although our analysis is extended via the single longitudinal mode lasers, this method can be also used in characterizing multi-mode lasers with large mode spacing. For Fabry-Perot (FP) laser, the mode spacing is usually larger than 100GHz. Therefore, this method might be suitable for FP lasers.

As the development of the techniques of the optical spectrum measurement, the methods for characterizing optoelectronic devices using optical spectrum analysis are also continuously developing. It becomes a simple auxiliary method in the Lab to measure the frequency response and chirp parameter by optical spectrum analysis.

\section{Optical spectrum analysis of the optical multi-beam injected semiconductor lasers}

External optical injection of semiconductor lasers changes the dynamic characteristics of the injected laser cavity and leads to many phenomena, such as injection locking, push-pull effects, chaos, and period doubling (Simpson et al., 1997). In previous works, different kinds of semiconductor optical injected lasers have been investigated (Hui et al., 1991; Chang et al., 2003; Zhang et al., 2007). However, the investigations were mainly concerned about single-beam injected lasers. As to the authors' knowledge, little work has been done to study the multi-beam injected lasers. In this section, we investigate the optical spectral characteristics and frequency responses of an FP laser subject to the external light injection from another FP laser (Zhu et al., 2008) and frequency-pushing effect in a dual-beam injection system ( $\mathrm{Li}$ et al., 2010).

\subsection{Enhanced modulation bandwidth of a Fabry-Perot semiconductor laser subject to light injection from another Fabry-Perot laser}

Fig.1.14 illustrates the typical optical spectra of the slave and injected master lasers. The FP mode spacings of the slave and the master FP lasers are 200 and $203 \mathrm{GHz}$, respectively. Therefore, the wavelength detuning is different for all FP modes. From Fig. 1.14, it can be seen that the mode spacing difference between the main FP modes of the two lasers are 9, 12 , and $15 \mathrm{GHz}$, respectively. It has been shown that the frequency response of an injection locked laser depends on both the injection optical power and wavelength detuning (Chrostowski, 2006). Therefore, we intuitively assume that a flat frequency response can be obtained after multimode light injection when the wavelength detunings and injection optical power are different for all FP modes. 


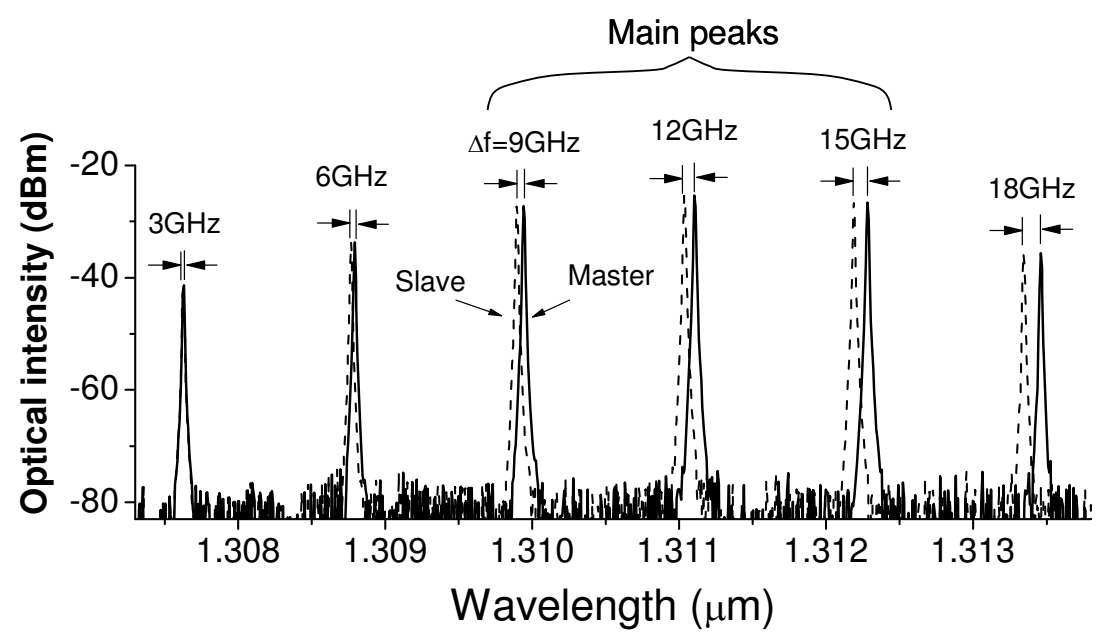

Fig. 1.14. Ideal optical spectra of the master and slave FP lasers. The mode spacing difference is $3 \mathrm{GHz}$

The optical injection operation efficiency of the two FP lasers is not always optimal. To achieve the best modulation bandwidth enhancement, we investigate the optimal conditions of the two FP lasers through the following experiments.

a. Wavelength matching conditions

The wavelength detuning of the FP modes can be adjusted by changing the bias current of the master and the slave lasers. Fig. 1.15 gives the experimental results when the mode spacing difference is $4 \mathrm{GHz}$. From the measured optical spectra shown in Fig. 1.15(a) and the inserted figures, it can be seen that for the main peaks the wavelength detuning are 25.5, $21.5,17.5,13.5,9.5$, and $5.5 \mathrm{GHz}$, respectively. Therefore, the wavelength detunings vary in a wide frequency range for different FP modes. From the measured frequency responses given in Fig. 1.15(b), it can be seen that both the 3-dB modulation bandwidth and the relaxation oscillation frequency increase by $10 \mathrm{GHz}$, and a very flat frequency response can be achieved within $18 \mathrm{GHz}$ after optical injection.

Fig. 1.16 shows the results when the mode spacings of the slave and master lasers are almost identical. The mode spacings of the master and slave lasers are 178.68 and $178.82 \mathrm{GHz}$. Since the mode spacing difference of two FP lasers is only $0.14 \mathrm{GHz}$, the wavelength detunings for different FP modes vary in a narrow frequency range. From Fig. 1.16(a), it can be seen that the optical spectra of all main peaks are very similar. Therefore, the frequency response improvement can happen in a narrow frequency range, even for higher injection optical power. As shown in Fig. 1.16(b), the resonant frequency increases by $2 \mathrm{GHz}$ after optical injection.

b. Central wavelength overlap

The central wavelength overlap can be adjusted by changing the bias current of the master laser. In the measurement, an EDFA and an attenuator are used to keep the injection optical power unchanged when adjusting the bias current of the master laser. From Fig. 1.17(a) it can be seen that the central wavelength are fully overlapped when the master laser is biased at $31.5 \mathrm{~mA}$. The measured frequency responses (Fig. 1.17(b)) show that the resonance 
frequency increases by $4 \mathrm{GHz}$. However, when the bias current of the master laser increases to $57 \mathrm{~mA}$, the central wavelengths become mismatched. In this case, the 3-dB modulation bandwidth increases slightly.

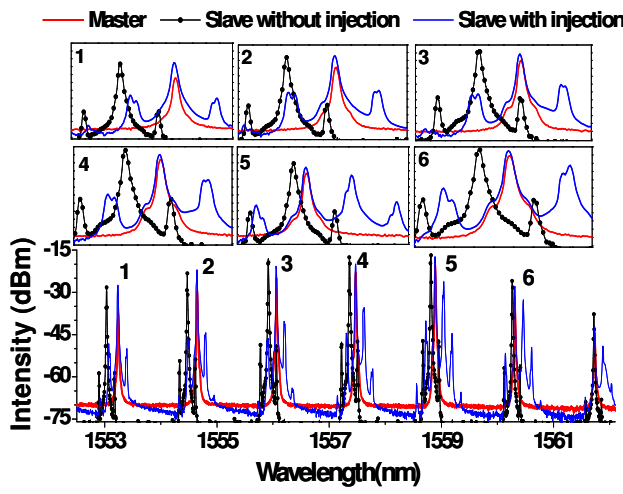

(a)

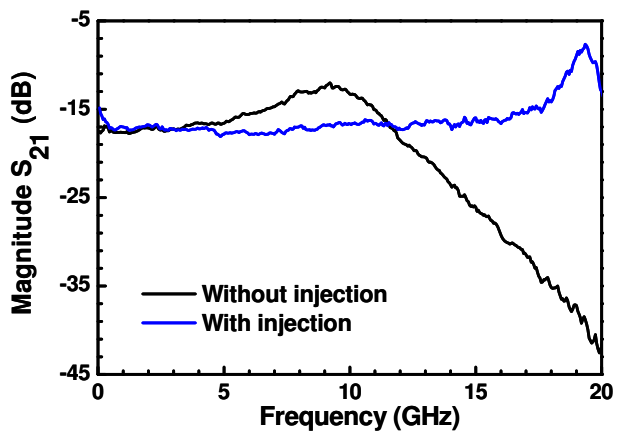

(b)

Fig. 1.15. (a) Measured optical spectra at the modulation frequency of $17 \mathrm{GHz}$. The insets show the higher resolution spectra. (b) Frequency responses of the slave laser, where the injection optical power is $8.3 \mathrm{~mW}$, and the mode spacing difference is $4 \mathrm{GHz}$

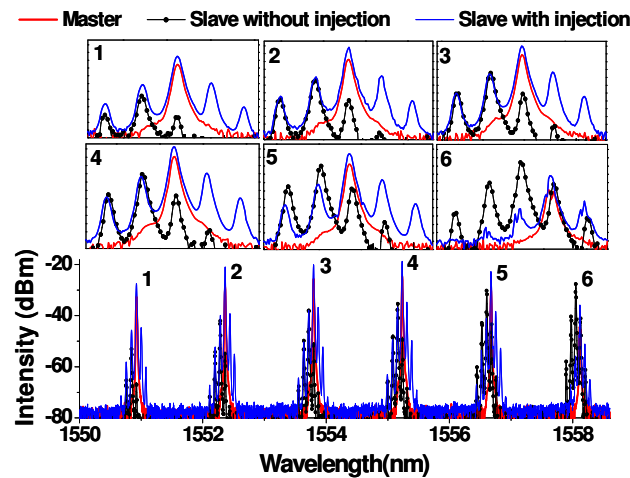

(a)

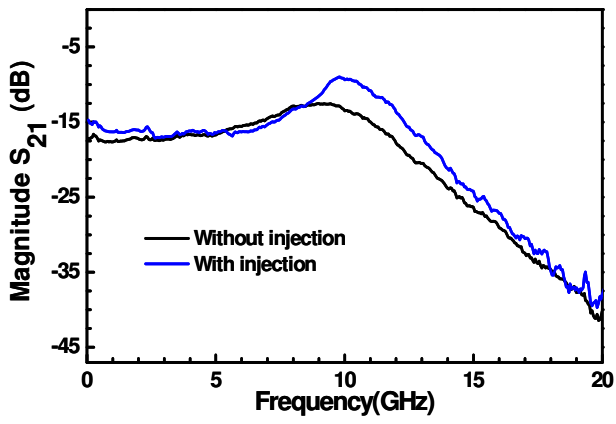

(b)

Fig. 1.16. (a) Measured optical spectra at the modulation frequency of $10 \mathrm{GHz}$. The insets show the higher resolution spectra. (b) Frequency response of the slave laser at different wavelength detuning, where the injection optical power is $3.6 \mathrm{~mW}$, and the mode spacing difference is $0.14 \mathrm{GHz}$

In addition to the above two main factors, the total optical injection power of the master laser and FP mode numbers can also affect the injection operation efficiency (Zhu et al., 2008). The optimal wavelength matching is mainly twofold: first, the close central wavelengths and more FP modes, and second, the mode spacing difference of several gigahertz. 


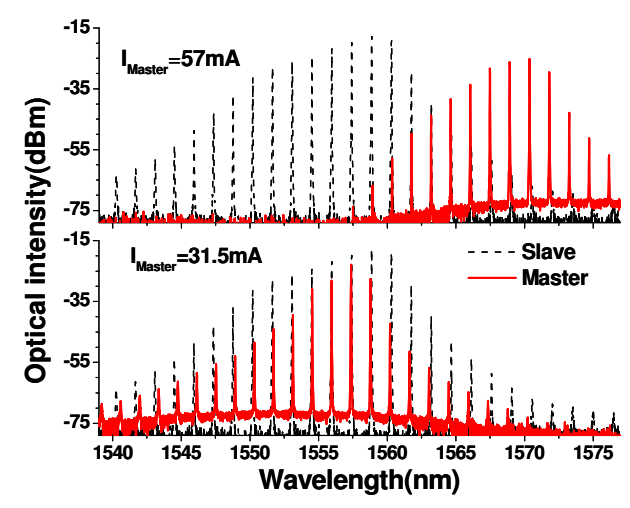

(a)

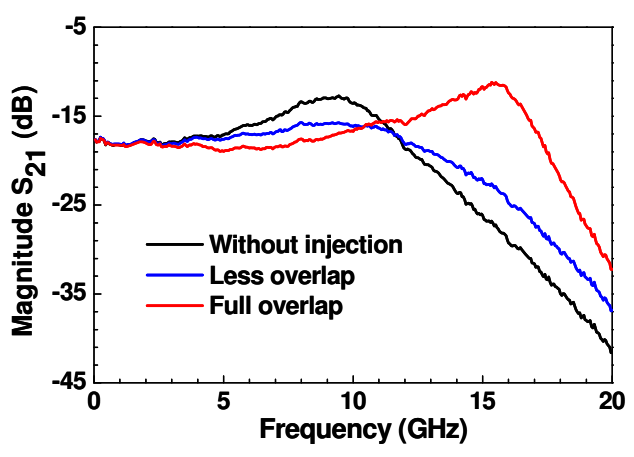

(b)

Fig. 1.17. (a) Measured optical spectra of the master laser biased at 57 and $31.5 \mathrm{~mA}$, compared with the measured spectrum of the slave laser. (b) Corresponding frequency responses of the slave laser, where the injection optical power is $3.9 \mathrm{~mW}$, and the mode spacing difference is $4 \mathrm{GHz}$

\subsection{Frequency-pushing effect in single-mode diode laser subject to external dual- beam injection}

A frequency-pushing effect can occur for semiconductor laser at positive detuning frequencies and higher injection ratios. The degree of pushing increases as the detuning frequency of the injection ratio increases or the external signal decreases (Simpson et al., 1997). Frequency convertor based on nearly degenerate four-wave mixing (NDFWM) in injection-locked semiconductor laser has been demonstrated for frequency conversion in optical communication systems, where two injection lasers are used. NDFWM in an injection-locked semiconductor laser has been studied in detail by Li and Petermann (Li \& Petermann, 1993). The NDFWM pump signal is provided by a semiconductor laser frequency-locked through optical injection of an external signal, and another external injection signal acts as the probe signal. Therefore, a conjugate signal is produced in the NDFWM process.

The influence of the frequency-pushing effect on dual-beam injection system can be observed intuitively in Fig. 1.18. Fig. 1.18(a) shows the free-running spectrum consisting of a single peak and weak relaxation resonance sidebands. The negative detuning frequency between the slave and the injection light (M1) is fixed out of the locking range as shown in Fig. 1.18(b). Then, another light (M2) is simultaneously injected at positive detuning frequency. As shown in Fig. 1.18(c), the spectrum consists of a slave signal and two regenerative amplified injected signals M1 and M2, and their detuning frequencies are both out of the locking range. For optical injection at positive detuning frequency, M2 pushes the slave signal to the negative detuning frequency. While the detuning frequency between M2 and the slave decreases, the degree of the frequency-pushing increases. Therefore, at a critical point, M1 falls into the locking range of the slave laser, i.e. the slave signal is locked to M1 as shown in Fig. 1.18(d). A converted signal C1 is observed at the red side of M1. As M2 continuously moves towards M1, the amplitude of C1 increases at first and then 
decreases as shown in Fig. 1.18(d)-(f). This means that the conversion efficiency has maximum (Li \& Petermann, 1993). In Fig. 1.18(g), the slave light jumps nearby of its freerunning frequency when M2 gets close to M1 because M1 and M2 are both out of the locking range again.

To get a deep insight of this process, we explain it on the viewpoint of medium-gain. Firstly we consider a single-beam injection system. Since the external injection light enhances the stimulated emission inside the cavity, the carrier population gets depleted faster, which results in a reduction of the gain and the carrier density in the laser cavity. This gain reduction induced by external light injection (blue line) is illustrated in Fig. 1.19 by showing the gain spectrum and the loss spectrum. Furthermore, this gain reduction through $\beta$ (the linewidth enhancement factor) changes the slave laser cavity frequency. The amount of cavity resonance shift is: $\Delta \mathcal{\lambda}_{\text {cav }}(N)=-\left(\lambda_{0}^{2} / 2 \pi c\right) \beta g \Delta N / 2$ (Zhao et al., 2006), where $g$ is the differential gain, $N$ is the carrier density and $c$ is the speed of light. Since $\Delta N$ is always negative, the cavity resonance is red-shifted and $\Delta \lambda_{\text {cav }}(N)$ is increased as the injection power increased.

Under the optical injection at positive detuning frequencies, the cavity resonance shift exhibits a frequency-pushing effect, which can be related to the medium-gain spectrum of the slave laser. For an injection wavelength far from the cavity resonance, the medium gain is small, and the amount of stimulated emission (regenerative amplified master light) caused by injection light is also small. Thus, the cavity resonance shift as well as the carrier density reduction is small. The injection light experiences more cavity gain as it getting closer to the cavity resonance under a fixed injection power, leading to a further reduced carrier density and a further red-shifted cavity resonance. In this process, the regenerative amplified master light is amplified by the cavity gain and the cavity resonance mode is suppressed. Finally, the slave laser gain is smaller than its threshold value, the master laser compensates this reduction and the slave laser is lasting at the master wavelength when it is injection locked. This process is illustrated in Fig. 1.19(a)-(d).

For optical injection at negative detuning frequencies (Fig. 1.19(e)), the cavity resonance shift exhibits a frequency pulling effect towards the injected light. As the frequency detuning between the maser and slave light is decreased at a critical point (Fig. 1.19(f)), the cavity resonance shifts automatically and continually to the frequency of the injection light until injection locking. It can be explained as follows: at the critical point (the margin of the locking range), the negative detuning injection light pulls the cavity resonance to the red side by a little amount, thus, the injection light can experience more cavity gain, which results in a further reducing of the carrier density and a more red-shifted cavity resonance. The red-shifted cavity resonance makes the injection light experience even more cavity gain. This process repeats automatically until the slave laser gain is smaller than its threshold value, leading to injection locking as shown in Fig. 1.19(f)-(h). The physically process in Fig. 1.19 is important in understanding the frequency-pushing effect in dual masters-slave system.

The gain and optical spectra in Fig. 1.20(a)-(f) correspond to the process in Fig. 1.18(b)-(g). The slave laser gets sufficient push when M2 moves closer (Fig. 1.20(b)), as explained in Fig. 1.19(a)-(c), and then it falls into the locking range of M1. Thus, the slave light is locked to the frequency of M1 just like the process illustrated in Fig. 1.19(f)-(h). NDFWM between the M2and injection-locked slave accounts for the converted signal C1 and C2 as shown in Fig. 1.20(c). When the converted signal $\mathrm{C} 1$ coincides with the red-shifted cavity resonance (amplified spontaneous emission), $\mathrm{C} 1$ is amplified by the cavity resonance as shown in Fig. 
1.20(d). This explains the maximum of conversion efficiency from Fig. 1.18(d) to Fig. 1.18(f). In Fig. 1.20(f), the injection signals are out of the locking range of the slave laser. The interaction between the injected lights and the slave gain is very weak. Thus, the slave laser is lasing near its free-running frequency.

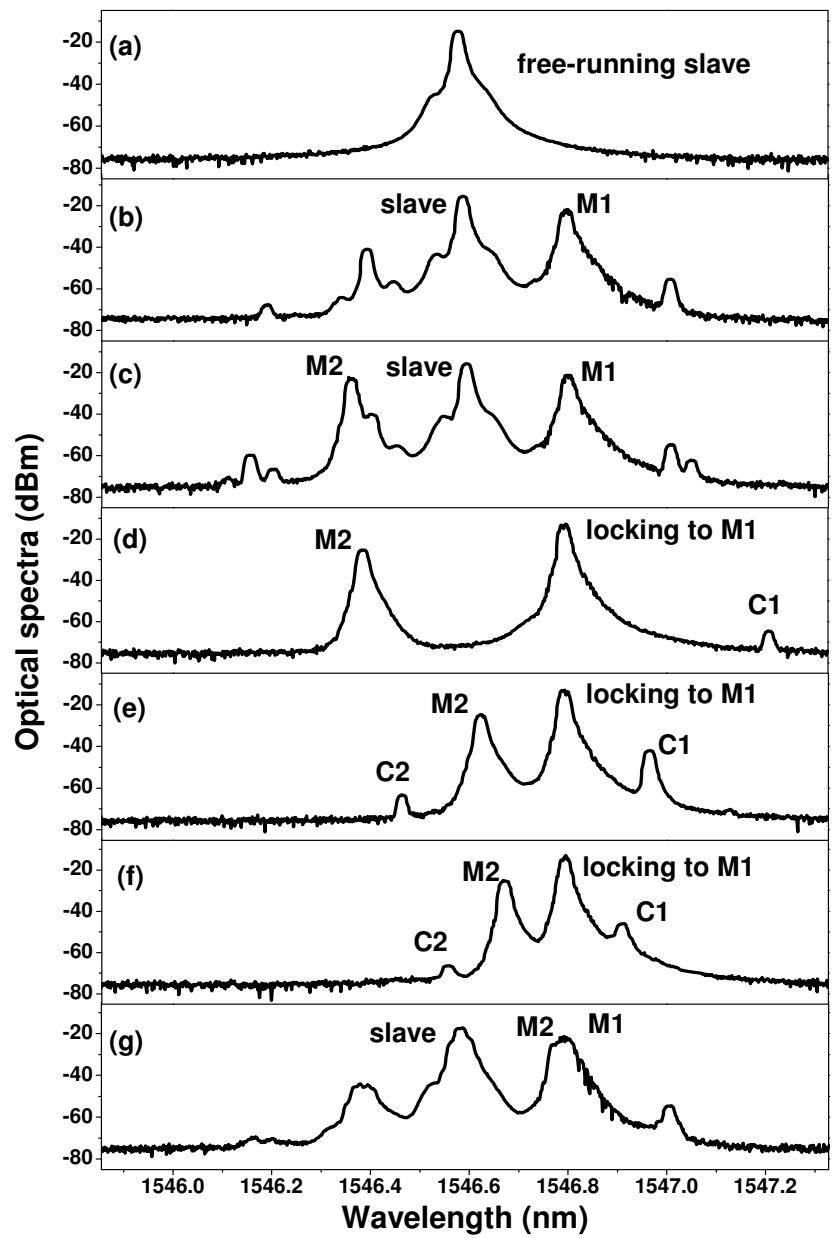

Fig. 1.18. Measured optical spectra in the process: M2 moves towards the slave signal from positive detuning frequency with a fixed optical power $(-15.37 \mathrm{~dB})$ while $\mathrm{M} 1$ is fixed at an operating point $(-13.73 \mathrm{~dB},-12.59 \mathrm{GHz})$

The further influence of the frequency-pushing effect on dual-beam injection system is shown in Fig. 1.21(a). Firstly, the conventional stable locking diagram is generated in a single master-beam injection system. The locking boundary is marked by open circles and dashed curve as shown in Fig. 1.21(a). Next, another injection signal from M2 injects simultaneously at a fixed operating point $(-10.12 \mathrm{~dB}, 12.08 \mathrm{GHz})$. A pushed locking range is observed in this case due to the frequency-pushing effect, whose boundary is indicated by 
red open triangles and dashed curve. The direction of pushing is indicated by a blue arrow, i.e. towards negative detuning frequency and low injection ratio. This is mainly because the injection of M2 pushes the slave frequency to the negative detuning frequency and suppresses its optical power by decreasing the slave gain.

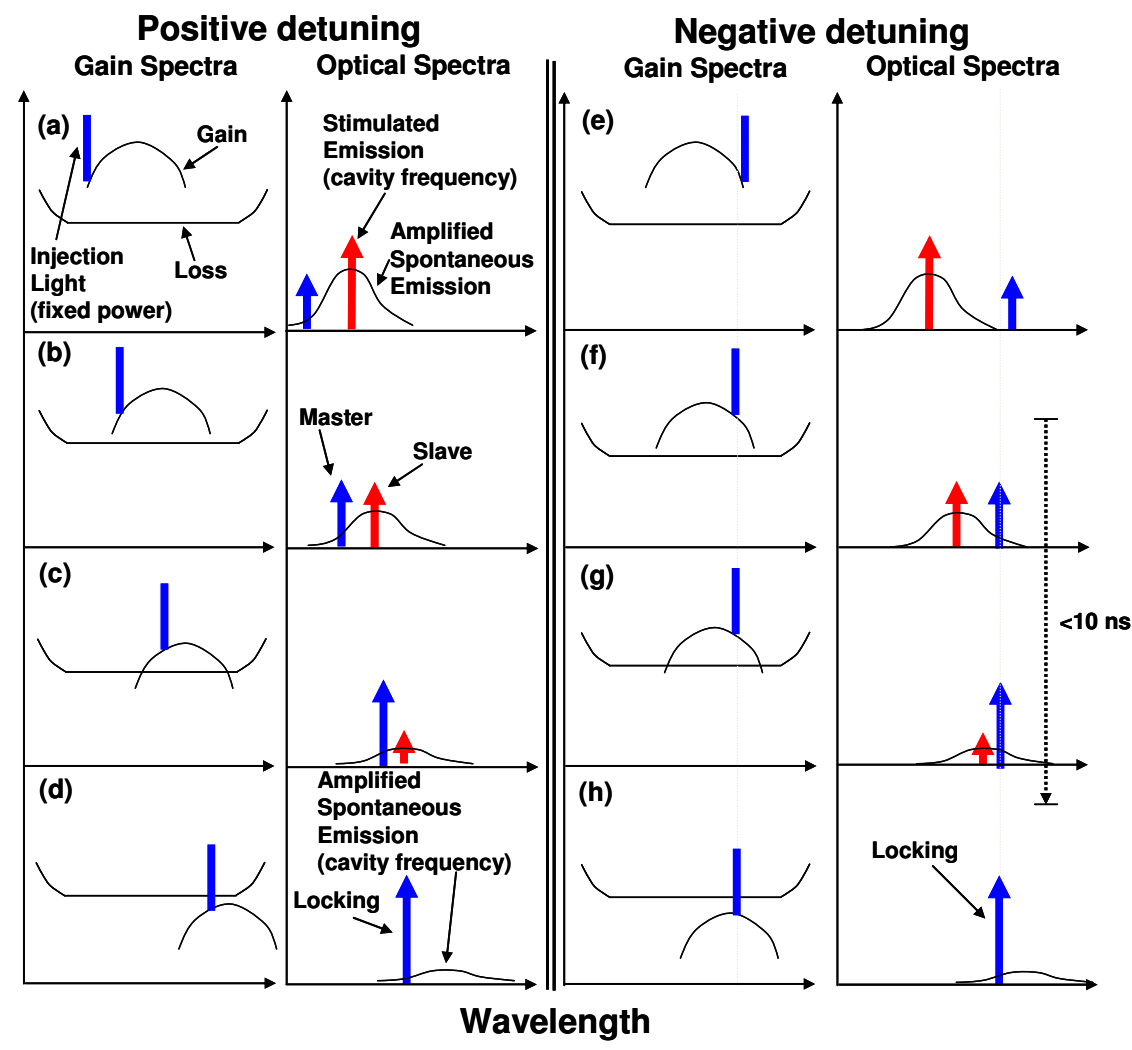

Fig. 1.19. Schematic showing the processes of frequency pushing and injection locking by medium-gain and optical spectra changing at positive and negative detuning frequencies with a fixed injection power in single-beam injection system

Fig. 1.21(b) gives the locking bandwidth as a function of power injection ratio under singlebeam injection (curve a) and dual-beam injection (curve b). It is obvious that the locking bandwidth varies with the power injection ratio. The variation of the locking bandwidth before and after injecting another beam is also shown in Fig. 1.21(b) (curve c= curve b-curve a). The horizontal dashed grey line as a reference indicates the identical locking bandwidths before and after injecting another beam. Under dual-beam injection, it can be seen that the frequency-pushing effect results in an increased locking bandwidth at most injection ratios except that around $-24 \mathrm{~dB}$. One can see that in Fig. 1.21(a) the negative detuning is limited to about $5 \mathrm{GHz}$ under moderate injection ratio in single-beam injection system. In the injectionlocked NDFWM process, the pump signal is provided by the slave laser which is injection locked to a master laser. The injection of the probe signal with moderate or high injection 
ratio can extend the negative detuning locking boundary, which allows a larger detuning frequency and lower injection ratio between the slave and M1.

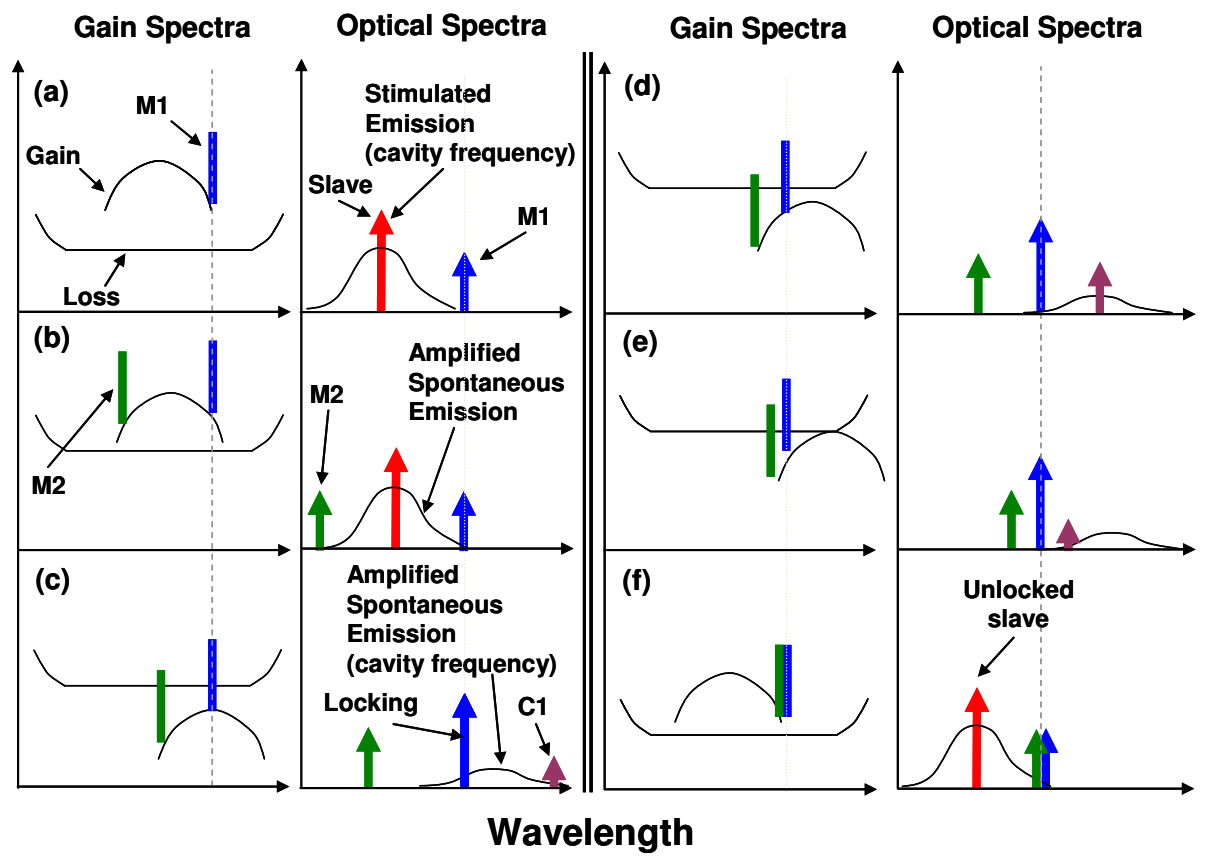

Fig. 1.20. Schematic showing the processes in Fig. 1.18 by medium-gain model

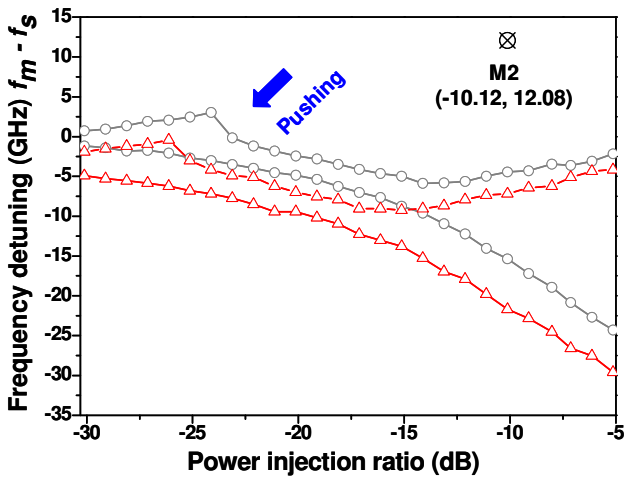

(a)

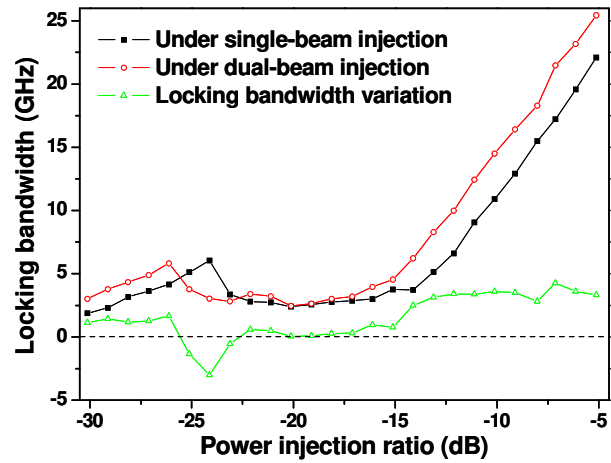

(b)

Fig. 1.21. (a) Experimental mapping of the stable locking range. The boundary of locking range in single-beam injection system is indicated by open circles and dash curve. The red open triangles and dash curve indicate the boundary of the pushed locking range by a fixed M2 (-10.12 dB, $12.08 \mathrm{GHz})$. (b) Locking bandwidth as a function of power injection ratio 


\section{The prospect of optical and electrical spectra analyses}

In the previous sections, optoelectronic device characterization in the electrical and optical domain has been extensively investigated. In this section we make a comparison between these two techniques and discuss their potential applications. Some conclusions could be made as follows:

1. The optical spectrum contains rich information of characteristics of optoelectronic devices. For example, the junction temperature can be obtained by measuring the output spectrum of a semiconductor laser driven by short pulses due to the close relationship between the output wavelength and the junction temperature. The frequency response of an optoelectronic device can also be estimated by analyzing the intensities of the optical spectrum sidebands. One of the main advantages of this technique is that there is no upper limit in measurement bandwidth compared to the methods based on electrical spectrum analysis, since no lightwave receiver is needed in the measurement. As the development of the spectrometry measurement techniques, the optical spectrum analysis has become a promising measurement technique in many microwave photonics labs over the world.

2. Although optical spectrum analysis has many advantages, its accuracy can not meet the requirements in some special applications due to the limited resolution of the OSA. The typical resolution is around several tens of picometers. By using the optical heterodyne technique, the optical spectra could be converted to intermediate frequencies in the electrical domain. Thus, the resolution of the optical spectrum analysis can be dramatically improved. For instance, the measured average linewidth of wave trains is narrower than $1 \mathrm{mHz}$, and the frequency response of an photodetector with ultra-wide bandwidth can also be precisely measured. It is also possible to analyze the real-time spectra of all types of wideband lightwave sources with the developed techniques.

3. Both the optical and electrical spectrum analyses play an important role in the optical generation of microwave techniques. A microwave signal can be generated in the optical domain by using optical heterodyning, in which two lightwaves with different wavelengths beat in a nonlinear optoelectronic device. This technique is able to generate a microwave signal with ultra high frequency (e.g. more than $100 \mathrm{GHz}$ ). Further more, the optical generated microwave signal can be distributed via optical fiber, so that it could greatly simplify the equipment requirement in applications such as the wireless communication system.

The amazing advantage of microwave photonics lies in the combination of the optical domain and electrical domain. There are still a lot of issues to be further investigated. For example, it is difficult to measure the optical spectrum with high resolution at a wide optical spectrum range simultaneously with good stability. In other words, the measurement resolution, spectral range and the system stability are difficult to be guaranteed simultaneously. The main factors, which have significant influence on the measurement results, are polarization state, phase and intensity noises of the light and so on. In summary, the combination of the analyses in optical and electrical domains is very promising for the measurement of optoelectronic devices, which is increasingly improved on the stage of rapid development.

\section{References}

Auracher F. \& Keil R. (1980). Method for measuring the RF modulation characteristics of MachZehnder-type modulators. Appl. Phys. Lett., Vol.36, No. 8, 626-629, ISSN 0003-6951. 
Baney, D. M.; Sorin, W. V.; \& Newton, S. A. (1994). High-frequency photodiode characterization using a filtered intensity noise technique. IEEE Photon. Technol. Lett., Vol. 6, No.10, (October, 1994) 1258-1260, ISSN 1041-1135

Bergano, N. S. (1988). Wavelength discriminator method for measuring dynamic chiro in DFB lasers. Electron. Lett., Vol. 24, No. 20, (September, 1988) 1296-1297, ISSN 00135194

Bjerkan L., Royset A., Hafskjaer L. \& Myhre D. (1996). Measurements of Laser Parameters for Simulation of High Speed Fiberoptic Systems. J. Lightwave Technol., 1996, Vol. 14, No. 5, 839-850, ISSN 0733-8724.

Chang, C.-H.; Chrostowski, L. \& Chang-Hasnain, C. J. (2003). Injection locking of VCSELs. IEEE J. Quantum Electron., Vol. 9, No. 5, (2003) 1386-1393, ISSN 1077-260X.

Chrostowski, L.; Zhao, X.; Chang-Hasnain C. J.; Shau, R.; Ortsiefer, M. \& Amann M.-C. (2006). 50-GHz optically injection-locked 1.55- $\mu \mathrm{m}$ VCSELs. IEEE Photon. Technol. Lett., Vol. 18, No. 2, (Jan. 2006) 1063-1066, ISSN 1041-1135.

G. Agrawal. (1985). Power spectrum of directly modulated single-mode semiconductor lasers: Chirp-induced fine structure. J. Quantum Electron., Vol. 21, No. 6, 680-686, ISSN 0018-9197.

Hawkins, R. T.; Jones, M. D.; Pepper, S. H. \& Goll, J. H. (1991). Comparison of fast photodetector response measurements by optical heterodyne and pulse response techniques. J. Lightwave Technol., Vol. 9, No. 10, (October, 1991) 1289-1294, ISSN 1289-1294

Henery E., Chusseau L. \& Lourtioz J. M. (1989). Frequency characterization of photodetectors by fabry-perot interferometry of modulated semiconductor lasers. Electron. Lett., Vol. 25, No. 1, 42-44, ISSN 0013-5194.

Hou, S.; Tucker, R. S. \& Koch, T. L. (1989). High-speed photodetector characterisation by delayed self-heterodyne method. Electron. Lett., Vol. 25, No. 24, (November, 1989) 1632-1634, ISSN 0013-5194

Huang, H. P.; Zhu, N. H. (2006). Small-signal power measuring technique for measuring the frequency response of electroabsorption modulators. IEEE Photon. Technol. Lett., Vol. 18, No. 23, (December, 2006) 2451-2453, ISSN 1041-1135

Hui, R.; D'Ottavi, A.; Mecozzi, A. \& Spano, P. (1991). Injection locking in distributed feedback semiconductor lasers. IEEE J. Quantum Electron., Vol. 27, No. 6, (June 1991) 1688-1695, ISSN 0018-9197.

Humphreyst, D. (1989). Integrated-optic system for high-speed photodetector bandwidth measurements. Electron. Lett., Vol. 25, No. 23, (November, 1989) 1555-1557, ISSN 0013-5194

Joseph, H. \& Sadot, D. (2004). A novel self-heterodyne method for combined temporal and spectral high-resolution measurement of wavelength transients in tunable lasers. IEEE Photon. Technol. Lett., Vol. 16, No. 8, (August, 2002) 1921-1923, ISSN 1041-1135

Kawanishi T., Kogo K., Oikawa S. \& Izutsu M. (2001). Direct measurement of chirp parameters of high-speed Mach-Zehnder-type optical modulators. Opt. Commun., 195:399-404, ISSN 0030-4018.

Kowalski B., Debeau J. \& Boittin R. A. (1999). Simple and novel method for measuring the chirp parameter of an intensity modulated light source. IEEE Photon. Technol. Lett., Vol. 11, No. 6, 700-702, ISSN 1041-1135. 
Kruger U. \& Kruger K. (1995). Simultaneous Measurement of the Linewidth, Linewidth Enhancement Factor and FM and AM Response of a Semiconductor Laser. J. Lightwave Technol., Vol. 13, No. 4, 592-597, ISSN 0733-8724.

Li, L. \& Petermann, K. (1993). Small-signal analysis of THz optical-frequency conversion in an injection-locked semiconductor laser. IEEE J. Quantum Electron., Vol. 29, No. 12, (Dec. 1993) 2988-2994, ISSN 0018-9197.

Li, W.; Zhu, N. H.; Wang, L. X.; Ke, J. H.; Chen, S. F.; Qi, X. Q.; Zhang, B. H. \&Xie, L. et al (2010). Frequency-Pushing Effect in Single-Mode Diode Laser Subject to External Dual-Beam Injection. IEEE J. Quantum Electron., Vol. 46, No. 5, (May. 2010) 796-803, ISSN 0018-9197.

Ohishi, T.; Ohtsuka, K.; Matsui, T. \& Ogata, H. (1989). Frequency characterisation of photodetectors by Fabry-Perot interferometry of modulated semiconductor lasers. Electron. Lett., Vol. 25, No. 1, (January, 1989) 42-44, ISSN 0013-5194

Oikawa S., Kawanishi T. \& Izutsu M. (2003). Measurement of chirp parameters and halfwave voltage of Mach-Zehnder-type modulators by using a small signal operation. IEEE Photon. Technology Lett., Vol. 15, 682-684, ISSN 1041-1135.

Petermann K. (1991). Laser diode modulation and noise. Kluwer Academic Publishers, ISBN 90277-2672-8.

Schimpe R., Bowers J. E. \& Koch T. L. (1986). Characterization of frequency response of 1.5 $\mu \mathrm{m}$ InGaAsP DFB laser diode and InGaAs pin photodiode by heterodyne measurement technique. Electron. Lett., Vol. 22, No. 9, 453-454, ISSN 0013-5194.

Shao, Y. \& Gallawa, R. L. (1986). Fiber bandwidth measurement using pulse spectrum analysis. Appl. Opt., Vol. 25, No. 7, (April, 1986) 1069-1071, ISSN 1559-128X

Shi Y., Yan L. S., \& Willner A. E. (2003). High-speed electrooptic modulator characterization using optical spectrum analysis. J. Lightwave Technol., Vol. 21, No. 10, 2358-2367, ISSN 0733-8724.

Simpson, T. B.; Liu, J. M.; Huang, K. F. \& Tai, K. (1997). Nonlinear dynamics induced by external optical injection in semiconductor lasers. Quantum Semiclass. Opt., Vol. 9, (May, 1997) 765-784, ISSN 1355-5111.

Villafranca A., Lazaro J. A., Salinas I. \&Garces I. (2005). Measurement of the linewidth enhancement factor in DFB lasers using a high-resolution optical spectrum analyzer. Photon. Technol. Lett., 2005, Vol. 17, No. 11, 2268-2270, ISSN 1041-1135.

Yan L. S., Shi Y., \& Willner A E. (2003). Chirp Measurement of Electro-Optic Modulators Using Simple Optical Spectrum Analysis. OFC' 03, paper MF58, ISBN 1-55752-7466.

Yan L. S., Shi Y., \& Willner A E. (2005). Graphical Solution for RF Half-Wave Voltage and Chirp Parameter of Electro-Optic Modulators Using Optical Spectrum Analysis. IEEE Photon. Technol. Lett., Vol. 17, No. 7, 1486-1488, ISSN 1041-1135.

Yu, Y. \& O'Dowd, R. (2002). Interpretation of wavelength switching effects of widely tunable lasers. IEEE Photon. Technol. Lett., Vol. 14, No. 10, (October, 2002) 1397-1399, ISSN 1041-1135

Zhang, B. H.; Zhu, N. H.; Han, H.; Ke, J. H.; Zhang, H. G.; Ren, M.; Li, W. \& Xie, L. (2009). Development of Swept Frequency Method for Measuring Frequency Response of Photodetectors Based on Harmonic Analysis. IEEE Photon. Technol. Lett., Vol. 21, No. 7, (April, 2009) 459-461, ISSN 1041-1135 
Zhang T., Zhu N. H., Zhang B. H. \& Zhang X. (2007). Measurement of chirp parameter and modulation index of a semiconductor laser based on optical spectrum analysis. IEEE Photon. Technol. Lett., Vol. 19, No. 4, 227-229, ISSN 1041-1135.

Zhang, X.; Zhu, N. H. ; Xie, L. \& Feng, B. X. (2007). A stabilized and tunable singlefrequency erbium-doped fiber ring laser employing external injection locking. $J$. Lightwave Technol., Vol. 25, No. 4, (Apr. 2007) 1027-1033, ISSN 0733-8724.

Zhao, X.; Change-Hasnain, C. J. Hofmann, W. \& Amann, M.-C. (2006). Modulation efficiency enhancement of $1.55-\mu \mathrm{m}$ injection-locked VCSELs. Proceeding of IEEE 20th int. Semiconductor laser conf. pp. 125-126, New York.

Zhu, N. H.; Li, W.; Wen, J. M.; Han, W.; Chen, W. \& Xie, L. (2008). Enhanced modulation bandwidth of a Fabry-Perot semiconductor laser subject to light injection from another Fabry-Perot laser. IEEE J.Quantum Electron., Vol. 44, No. 6, (June 2008) 528535, ISSN 0018-9197.

Zhu, N. H.; Wen, J. M.; San, H. S.; Huang, H. P.; Zhao, L. J. \& Wang, W. (2006). Improved self-heterodyne method for measuring frequency responses of photodetectors. IEEE Quantum Electron., Vol. 42, No. 3, (March, 2006) 241-248, ISSN 0018-9197 


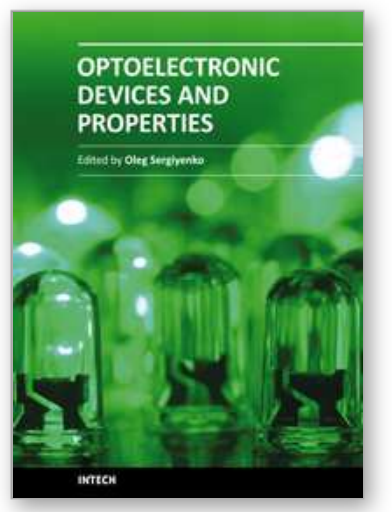

\section{Optoelectronic Devices and Properties}

Edited by Prof. Oleg Sergiyenko

ISBN 978-953-307-204-3

Hard cover, 660 pages

Publisher InTech

Published online 19, April, 2011

Published in print edition April, 2011

Optoelectronic devices impact many areas of society, from simple household appliances and multimedia systems to communications, computing, spatial scanning, optical monitoring, 3D measurements and medical instruments. This is the most complete book about optoelectromechanic systems and semiconductor optoelectronic devices; it provides an accessible, well-organized overview of optoelectronic devices and properties that emphasizes basic principles.

\section{How to reference}

In order to correctly reference this scholarly work, feel free to copy and paste the following:

Ning Hua Zhu, Wei Chen, Wei Li, Li Xian Wang, Xiao Qiong Qi and Bang Hong Zhang (2011). Optical and Electrical Spectrum Analysis of Optoelectronic Devices, Optoelectronic Devices and Properties, Prof. Oleg Sergiyenko (Ed.), ISBN: 978-953-307-204-3, InTech, Available from:

http://www.intechopen.com/books/optoelectronic-devices-and-properties/optical-and-electrical-spectrumanalysis-of-optoelectronic-devices

\section{INTECH}

open science | open minds

\section{InTech Europe}

University Campus STeP Ri

Slavka Krautzeka 83/A

51000 Rijeka, Croatia

Phone: +385 (51) 770447

Fax: +385 (51) 686166

www.intechopen.com

\section{InTech China}

Unit 405, Office Block, Hotel Equatorial Shanghai

No.65, Yan An Road (West), Shanghai, 200040, China

中国上海市延安西路65号上海国际贵都大饭店办公楼 405 单元

Phone: +86-21-62489820

Fax: +86-21-62489821 
(C) 2011 The Author(s). Licensee IntechOpen. This chapter is distributed under the terms of the Creative Commons Attribution-NonCommercialShareAlike-3.0 License, which permits use, distribution and reproduction for non-commercial purposes, provided the original is properly cited and derivative works building on this content are distributed under the same license. 